\title{
Higgs boson pair production merged to one jet
}

\author{
Philipp Maierhöfer and Andreas Papaefstathiou \\ Institut für Theoretische Physik, Universität Zürich, \\ Zürich, Switzerland \\ E-mail: philipp@physik.uzh.ch, andreasp@physik.uzh.ch
}

ABStRaCT: We develop a Monte Carlo event generator for Higgs Boson pair production merged to exact one-jet matrix elements. The matrix elements are generated with OpenLoops and event generation is performed with the HERWIG++ general-purpose event generator. This allows us to simulate fully-exclusive hadronic final states with accurate description of the kinematics of the leading jet in conjunction with a parton shower. We use the implementation to examine in detail the systematic uncertainties which result from the merging procedure. We assess the magnitude of the impact of the merging on experimental searches of Standard Model di-Higgs production that aim to constrain the Higgs boson self-coupling. We find that the use of a merged sample can reduce theoretical systematic uncertainties in the efficiencies of cuts on certain observables. This constitutes the most accurate simulation of the process available to date. The Monte Carlo event generator developed for this project is available as an add-on to the HERWIG++ event generator at http://www.physik.uzh.ch/data/openloops/download/projects/hhmerge/.

Keywords: Monte Carlo Simulations, Hadronic Colliders

ARXIV EPRINT: 1401.0007 


\section{Contents}

1 Introduction 1

1.1 Context and introductory remarks 1

$\begin{array}{ll}1.2 & \text { Di-Higgs production at higher orders }\end{array}$

2 OpenLoops and matrix elements 3

2.1 The OpenLoops matrix element generator 3

2.2 Higgs pair production matrix elements 3

3 Merging $\quad 4$

3.1 Merging methods 4

$\begin{array}{lll}3.2 & \text { Systematic uncertainties } & 6\end{array}$

4 Phenomenological implications $\quad 11$

5 Conclusions $\quad 15$

\section{Introduction}

\subsection{Context and introductory remarks}

The Large Hadron Collider ATLAS and CMS experiments confirmed the existence of a scalar particle consistent with the Standard Model (SM) Higgs Boson [1-5]. However the quest for understanding the mechanism behind the electroweak symmetry breaking (EWSB) does not end with the discovery of the Higgs Boson; measuring its couplings to the SM fields is an important and long-term task that the ATLAS and CMS experiments, as well as future collider experiments, are expected to undertake. It is crucial, moreover, to determine whether the realisation of the mechanism of the EWSB is indeed SM-like. This can be investigated by examining the Higgs potential which, after EWSB in the minimal prescription, can be written as

$$
\mathcal{V}(h)=\frac{1}{2} m_{h}^{2} h^{2}+\lambda_{h h h} v h^{3}+\frac{1}{4} \lambda_{h h h h} h^{4} .
$$

Within the SM we have $\lambda_{h h h}^{\mathrm{SM}}=\lambda_{h h h h}^{\mathrm{SM}}=m_{h}^{2} /\left(2 v^{2}\right) \simeq 0.13$ for a Higgs boson mass of $m_{h} \simeq 125 \mathrm{GeV}$. The discovery of the Higgs boson only indicates the size of the curvature of the potential around the local minimum, coming from the quadratic term. To confirm the form of the potential, the measurement of higher-order terms is necessary. At the LHC, these terms can be probed directly via double or triple Higgs Boson production. The tiny cross section for triple Higgs production makes it impossible to perform any meaningful measurement in the foreseeable future, even during the full life-time of the 

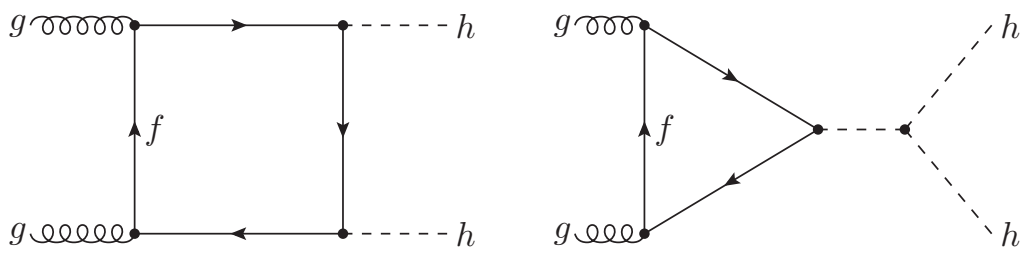

Figure 1. The Higgs pair production diagrams contributing to the gluon fusion process at LO are shown for a generic fermion $f$.

LHC $[6,7]$. Higgs Boson pair production, on the other hand, is certainly challenging but not impossible to observe at the LHC. Interesting phenomenological studies were performed more than 10 years ago [8-13] and more recently, owing to the discovery of the Higgs Boson as well as the development of boosted jet techniques, the subject has undergone a lively rejuvenation [14-28].

Despite the fact that several interesting and in-depth phenomenological studies of inclusive Higgs Boson pair production at the LHC $(p p \rightarrow h h+X)$ have been performed, the Monte Carlo event simulation of the process has relied so far only on leading-order matrix elements with the addition of parton showers to simulate the extra QCD radiation. ${ }^{1}$ Exceptions to this are two recent studies which examined the exclusive one- and two-jet channels in the full theory, with the full top mass dependence, (i.e. $p p \rightarrow h h j+X$ and $p p \rightarrow h h j j+X)$ and contrasted these to results obtained in the effective theory [16, 30]. It is important to stress, however, that the kinematical properties of inclusive final states can be substantially altered by the inclusion of higher-order matrix elements. This is especially true in the inclusive $h h+X$ process, which is predominantly gluon-gluon initiated, and hence is inevitably accompanied by a copious amount of QCD radiation. Thus, the accuracy, and hence reliability, of the kinematics of inclusive di-Higgs searches will certainly benefit from the inclusion of the exact real-emission higher-order matrix elements.

\subsection{Di-Higgs production at higher orders}

The $p p \rightarrow h h+X$ process at hadron colliders is loop-induced at leading order, proceeding via a heavy quark loop. The leading-order gluon fusion diagrams are shown in figure 1 . Evidently, a next-to-leading order calculation would involve, among others, diagrams with two loops that involve heavy fermions, and hence two mass scales (the fermion mass and the Higgs Boson mass). Such diagrams currently lie at the frontier of higher-order loop calculations. Consequently, this impedes the implementation of a matched next-to-leading order (NLO) plus shower simulation.

The effective theory approximation in the heavy top mass limit that has been employed in single Higgs boson production has been shown to be insufficient to describe the kinematics of the $h h$ process, both at leading order [22, 31], and at higher orders [16, 30]. Nevertheless, inclusive NLO [9, 32] and NNLO cross section calculations [20, 21] have

\footnotetext{
${ }^{1}$ During the final stages of preparation of this article, a similar study has appeared in ref. [29]. Here we provide a completely independent implementation, both in terms of the merging and the production frameworks employed.
} 
been performed in the effective theory approximation, giving an estimate of the size of the higher-order corrections in the full theory.

Thus, in the absence of the full NLO calculation, the best one can do is merge samples of different jet multiplicities in a consistent way, carefully avoiding any issues that may arise due to double-counting or phase space region mismatch. Such simulations have been shown to reliably describe the kinematical properties of experimental data (see for example the relevant experimental CMS [33] and ATLAS [34] analyses), modulo the correct normalisation taken from higher-order cross section calculations. Here we perform such a merging of samples, including the full top and bottom mass dependence in the fermion loops of figure 1, as well as the higher-order real emission diagrams which we examine below.

This paper is organised in the following way: in section 2 we briefly describe the OpenLoops generator for one-loop matrix elements and provide cross sections for the various contributing exclusive channels. In section 3 we present results and examine the systematic uncertainties associated with the merging prescription, and in section 4 we investigate the phenomenological implications of including the merged higher-order matrix elements. We present our conclusions in section 5 .

\section{OpenLoops and matrix elements}

\subsection{The OpenLoops matrix element generator}

The OpenLoops generator is based on the open-loops algorithm [35] for the efficient evaluation of one-loop matrix elements. The algorithm employs a numerical recursion to construct the loop momentum dependence of the numerator of loop amplitudes combined with tensor integral reduction. The tensor integrals are computed by the Collier library, which implements the Denner-Dittmaier reduction procedure for the numerically stable evaluation of tensor integrals [36, 37] and the scalar integrals of ref. [38].

Incidentally, using tensor integrals allows for a high degree of optimisation through caching, since the integrals can be shared across different Feynman diagrams for all helicity and colour configurations. For on-shell reduction approaches this is only possible when the loop amplitude is interfered with a tree amplitude [35] and therefore not in calculations of loop induced processes, like the one presented here.

\subsection{Higgs pair production matrix elements}

Like in the case of single Higgs boson production, the $h h$ production cross section at hadron colliders is dominated by the gluon fusion channels. For a more detailed dissection of the leading order cross section, we refer the reader to section 2 of ref. [18].

The classes of higher-order real emission diagrams that we include in our calculation are shown in figure 2. The real emission process also contains diagrams with $q g, \bar{q} g$ and $q \bar{q}$ initial states which are subdominant but non-negligible and must be included for consistent merging since the parton shower introduces $g \rightarrow q \bar{q}$ splittings on the initial state gluons of the 0-jet matrix elements [39]. It is important to note that the diagrams that involve radiation from the heavy quark loop, are not included in any limit in the parton shower 

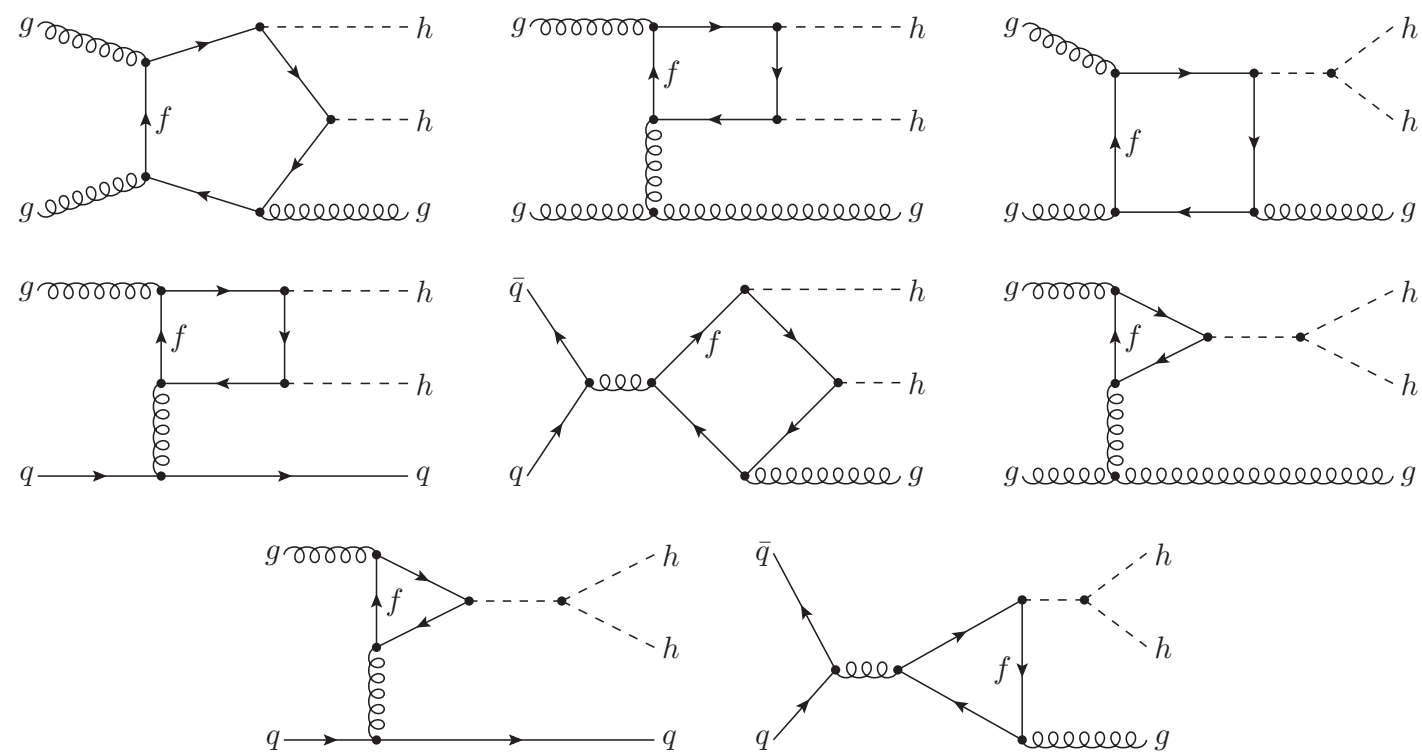

Figure 2. Diagram classes which contribute to Higgs boson pair production in association with one extra parton are shown for a generic fermion $f$ running in the loop.

Monte Carlos with which we merge with. Hence, an intrinsic assumption of the merging procedure is that these diagrams are sub-dominant with respect to the initial state radiation in the parton shower-dominated regime.

The OpenLoops process libraries to compute matrix elements have been interfaced with HERWIG++. These can be used stand-alone, i.e. without the merging, to perform studies of leading-order $h h$ production, or $h h+j$ production. In table 1 we present the cross sections for the different sub-processes contributing to $p p \rightarrow h h j+X$, where $j$ is an associated parton. ${ }^{2}$ Here, and throughout this paper, we use the 4-flavour MSTW2008nlo $68 \%$ confidence level parton density functions [41-43]. Obviously, even with the relatively high $p_{\perp}$ cut of $60 \mathrm{GeV}$, the real emission sub-processes possess a cross section that is comparable to the leading order gluon fusion process. This is an indication that they are indeed significant and have to be considered for an accurate description of the kinematics, even in an inclusive $h h+X$ analysis.

\section{Merging}

\subsection{Merging methods}

In order to obtain a realistic simulation of processes involving associated high- $p_{T}$ jet production, e.g. W/Z/Higgs+jets, the parton shower approximation for the generation of soft and collinear QCD radiation must be supplemented by high multiplicity leading-order matrix elements. Matrix element-parton shower merging schemes, such as the so-called MLM [44-46] and CKKW [44, 45, 47-50] methods, have been developed for this purpose. These methods work by partitioning phase space, by means of a jet algorithm, such that

\footnotetext{
${ }^{2}$ The HERWIG++ implementation has been crossed-check against the SHERPA event generator [40].
} 


\begin{tabular}{|c|c|c|c|c|c|}
\hline Process & $g g \rightarrow h h$ & $g g \rightarrow h h g$ & $g q \rightarrow h h q$ & $g \bar{q} \rightarrow h h \bar{q}$ & $q \bar{q} \rightarrow h h g$ \\
\hline$\sigma(14 \mathrm{TeV})[\mathrm{fb}]$ & $26.2(1)$ & $9.5(1)$ & $1.80(2)$ & $0.411(6)$ & $0.062(1)$ \\
\hline$\sigma(33 \mathrm{TeV})[\mathrm{fb}]$ & $145(3)$ & $70.2(9)$ & $10.0(1)$ & $3.39(5)$ & $0.206(3)$ \\
\hline$\sigma(100 \mathrm{TeV})[\mathrm{fb}]$ & $883(5)$ & $555(7)$ & $60.6(9)$ & $27.1(4)$ & $0.79(1)$ \\
\hline
\end{tabular}

Table 1. Cross sections for the partonic $p p \rightarrow h h+X$ and for the sub-processes contributing to $p p \rightarrow h h j+X$ at 14,33 and $100 \mathrm{TeV}$. For the case of real emission, a cut of $p_{\perp}>60 \mathrm{GeV}$ was placed on the associated parton. The factorisation/renormalisation scales were both fixed to $\mu=m_{h}+p_{\perp, j}$, where $p_{\perp, j}$ is the transverse momentum of the associated parton in the centre of mass frame.

the distribution of jets corresponds to that of the partons in the matrix elements, while the distribution of radiation inside the jets is appropriately developed by the shower. In addition, both the MLM and CKKW algorithms augment the distribution of radiation in the matrix element region with Sudakov suppression effects, not present in the matrix elements themselves, thus smoothing the transition from one radiation pattern to another at the phase space partition. ${ }^{34}$

HERWIG++ [55-58] includes an implementation of the MLM merging scheme. The current version of the merging algorithm has been validated against its FORTRAN HERWIG [59] counterpart for several processes. For the purposes of this project, the implementation has undergone minor modifications, to accommodate the use of internally-generated matrix elements. We use this algorithm in conjunction with the parton shower in order to merge the two. We fix the factorisation and renormalization scales to be equal, $\mu_{F}=\mu_{R}=\mu=\nu\left(m_{h}+p_{\perp}^{h h}\right)$, where $\nu$ is a parameter which we vary, $m_{h}$ and $p_{\perp}^{h h}$ are the Higgs boson mass and the transverse momentum (as defined in the centre-of-mass frame of the hard process) of the Higgs boson pair respectively. Note that for the LO $h h$ process, $p_{\perp}^{h h}=0$ and hence this implies that $\mu=\nu m_{h}$ for all, even showered, LO samples. We call the merging scale $E_{\text {Tclus }}$, inspired by the way the MLM method is implemented in the HERWIG++ generator. We call the lowest-order sample ' 0 -jet' and the sample including one real emission ' 1 -jet'. Broadly speaking, after showering is performed in HERWIG++, the MLM method will effectively veto all events in the '0-jet' sample that contain a jet with transverse momentum larger than $E_{\text {Tclus }}$. This will result in what we will call the '0-jet exclusive' sample. In the showered '1-jet' sample the MLM algorithm will effectively veto any events with jets that have not "matched" the given extra parton produced in association with the Higgs boson pair, as well as events that contain jets harder than the 'matched' jet. The resulting sample is called ' 1 -jet inclusive', meaning it contains no 0 -jet contributions

\footnotetext{
${ }^{3}$ For a full, comparative description of the available schemes, see ref. [46].

${ }^{4}$ It is also conceivable, at least in the case of one extra associated parton, to perform a simulation with the MC@NLO or POWHEG matching prescriptions, with an arbitrary virtual contribution which can be set to zero [51-54].

${ }^{5}$ The term 'matched' in the MLM prescription refers to whether a jet is found to be within a certain distance $\Delta R$, from a given hard parton that appears in the pre-showered event. By default this is taken to be $1.5 \times R_{\text {clus }}$, where the $R_{\text {clus }}$ is the clustering cone size used in the merging.
} 
but contains jets coming from the shower, of lower $p_{\perp}$ than the matrix element parton. For more details on the algorithm see, for example, [46].

Recently it has been shown [60] that the merging of samples of different multiplicities may result in kinks due to the presence of a significant mismatch in the description of extra emissions between the parton shower and the matrix element calculations in the region chosen for merging. In ref. [60], it was suggested that one can obtain smooth matching by the use of a smoothing ' $D$-function' that contains two scales instead of a single scale. Sudakov reweighting was also used to achieve an even smoother matching. Here we perform a variant of the former method: we generate a merging scale randomly in a given interval according to a given distribution. This merging 'range' is then characterised by two scales, an 'average' merging scale $\bar{E}_{\text {Tclus }}$ and a 'variation' scale $\epsilon_{\text {clus }}$. The merging scale is then randomly chosen on an event-by-event basis using two different 'schemes'. The first scheme uses a Sine function ('Sinusoidal'),

$$
E_{\text {Tclus }}=\frac{2 \epsilon_{\text {clus }}}{\pi} \sin ^{-1}(2 x-1)+\bar{E}_{\text {Tclus }}
$$

and the second a linear function ('Uniform'),

$$
E_{\text {Tclus }}=(2 x-1) \epsilon_{\text {clus }}+\bar{E}_{\text {Tclus }},
$$

where $x \in[0,1]$ is a uniform random number. The effect of these schemes is to smooth out the unphysical discontinuities, resulting in a continuous merging of the shower and matrix element descriptions.

To further improve the merging between the two samples, we perform ' $\alpha_{S}$-reweighting' of the 1-jet matrix elements according to (schematically)

$$
|\mathcal{M}|^{2} \rightarrow|\mathcal{M}|^{2} \frac{\alpha_{S}\left[\left(p_{\perp}^{h h}\right)^{2}\right]}{\alpha_{S}\left(\mu^{2}\right)}
$$

where $p_{\perp}^{h h}$ is the transverse momentum of the Higgs boson pair as defined in the centreof-mass frame of the hard process (or the transverse momentum of the associated extra parton) and $\mu$ is the renormalization scale. This is to accommodate the difference between the scale that the shower uses in the calculation of the strong coupling constant $\alpha_{S}$ wrt. the scale used in the matrix elements. In practice the effect of the reweighting is small, especially in comparison to the uncertainties arising from variations of $\mu$ and the merging scale parameters. All of the results in the rest of the paper include $\alpha_{S}$-reweighting.

\subsection{Systematic uncertainties}

In what follows we present results obtained at parton level, using the Rivet analysis framework version 1.8.3 [61] and the anti- $k_{T}$ algorithm [62] with $R=0.4$. In all the calculations, the Higgs boson mass was chosen to be $m_{h}=125 \mathrm{GeV}$ and the top quark and bottom quark masses to be $174.2 \mathrm{GeV}$ and $4.7 \mathrm{GeV}$ respectively, with all the widths set to zero. The renormalization and factorisation scales were both set to equal $\mu: \mu_{F}=\mu_{R}=\mu$.

We first examine the effect of the two schemes suggested to facilitate smooth merging, 'Sinusoidal' and 'Uniform', for $E_{\text {TClus }}=60 \mathrm{GeV}$ and $\epsilon_{\text {clus }}=30 \mathrm{GeV}$. We compare to the 

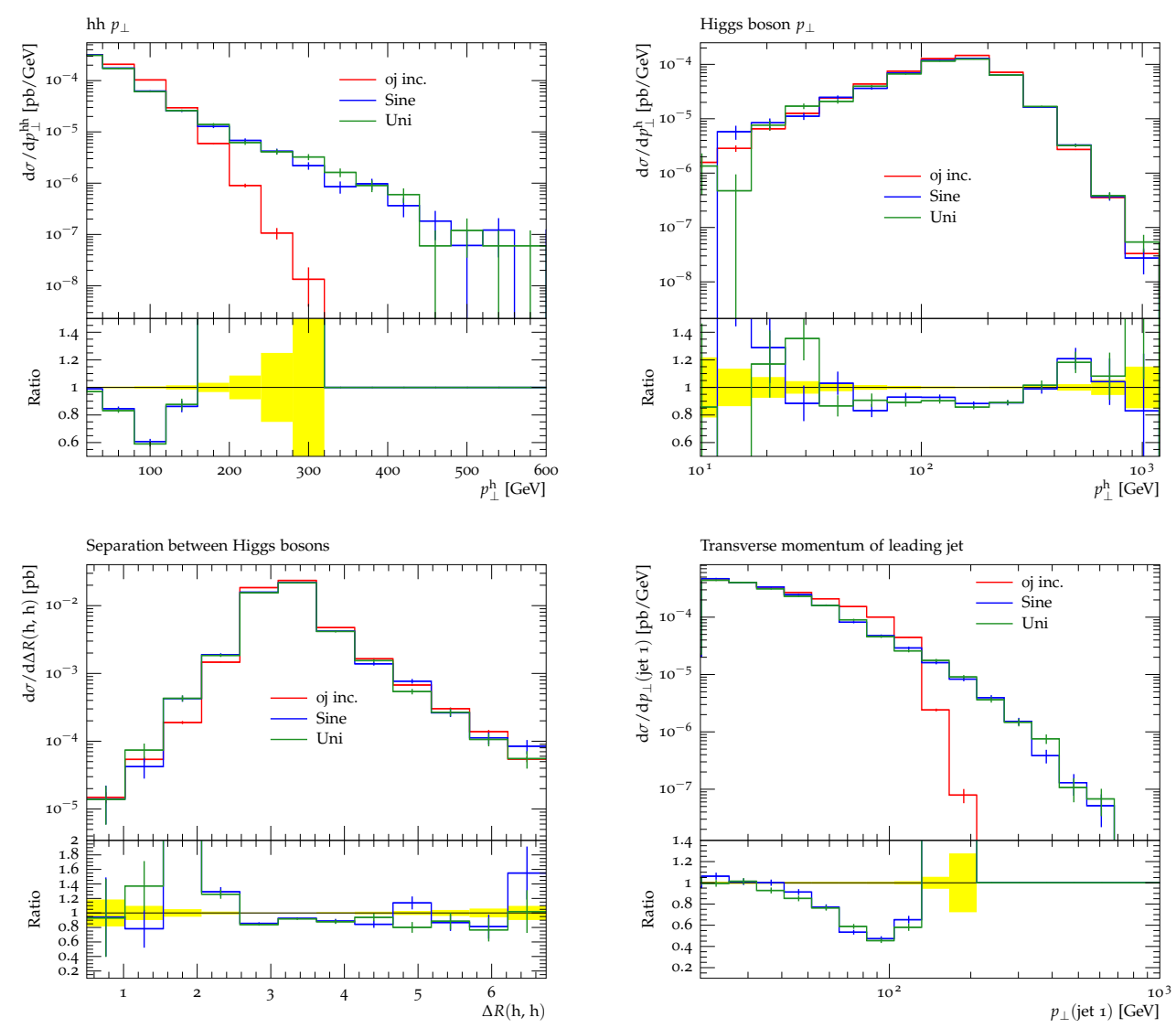

Figure 3. The transverse momentum of the di-Higgs system and the transverse momentum of a Higgs boson, $p_{\perp}^{h h}$ and $p_{\perp}^{h}$ respectively (top), the distance between the two Higgs bosons, $\Delta R(h, h)$, and the $p_{\perp}$ of the leading jet (bottom). A comparison between the two different smoothing schemes, 'Sinusoidal' and 'Uniform' is shown. The clustering parameters were set to $\bar{E}_{\text {Tclus }}=60 \mathrm{GeV}$, $\epsilon_{\text {clus }}=30 \mathrm{GeV}$ in both cases. We also show the un-merged sample ('0j inc.') with $\mu=m_{h}$, with respect to which the ratio sub-plot is taken.

purely showered LO sample ('un-merged') with $\mu=m_{h}$. In figure 3 we show the transverse momentum of the di-Higgs system and the transverse momentum of a Higgs boson, $p_{\perp}^{h h}$ and $p_{\perp}^{h}$ respectively, the distance between the two Higgs bosons, $\Delta R(h, h)$ and the $p_{\perp}$ of the leading jet. It is evident by examining the plots that the 'Uniform' scheme provides stronger smoothing than the 'Sinusoidal' scheme. Considering that the disagreement between the un-merged sample and the merged samples in the merging regions is large, we suggest the use the 'Uniform' scheme for merging in the $h h$ process and we employ this in the rest of this study.

In figure 4 we examine the effect of the variation of the scale $\mu$ on various distributions for the merged samples. We vary the scale $\mu$ between $\mu=m_{h}+p_{\perp}^{h h}$ and $\mu=4\left(m_{h}+\right.$ $\left.p_{\perp}^{h h}\right)$, while fixing $\bar{E}_{\text {Tclus }}=60 \mathrm{GeV}$ and $\epsilon_{\text {clus }}=30 \mathrm{GeV}$. For comparison, we also show the equivalent un-merged scale variation between $\mu=m_{h}$ and $\mu=4 m_{h}$. It is evident that, modulo normalisation differences originating from the scale variation, the general shapes of the distributions exhibit reasonable stability over the range of the chosen scales for 

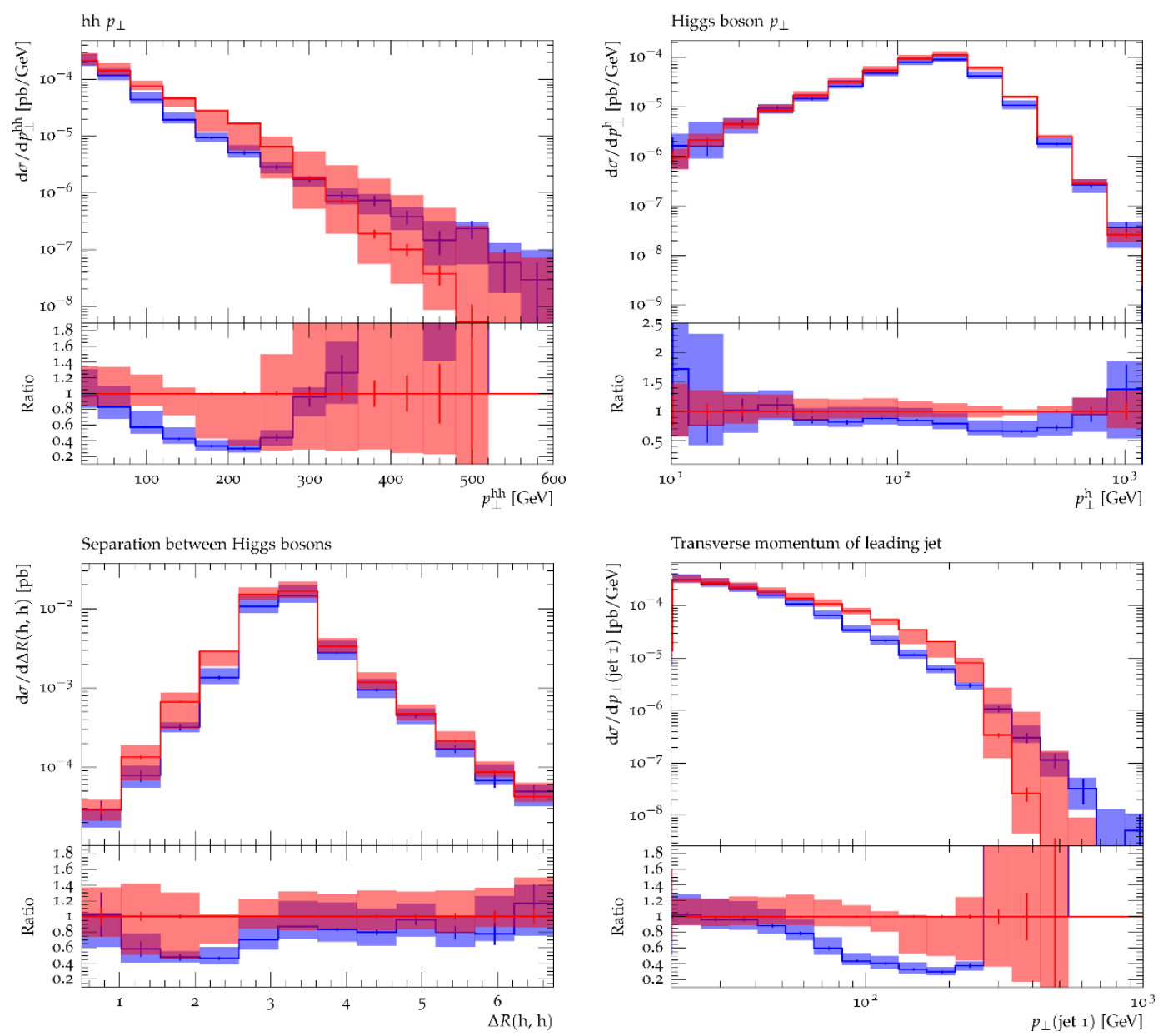

Figure 4. The transverse momentum of the di-Higgs system and the transverse momentum of a Higgs boson, $p_{\perp}^{h h}$ and $p_{\perp}^{h}$ respectively (top), the distance between the two Higgs bosons, $\Delta R(h, h)$, and the $p_{\perp}$ of the leading jet (bottom). The merged samples are shown in blue, with the blue line corresponding to $\mu=2\left(m_{h}+p_{\perp}^{h h}\right)$ and the un-merged samples are shown in red, with the red line corresponding to $\mu=2 m_{h}$. The bands show the envelope of scale variations between $\mu=m_{h}+p_{\perp}^{h h}$ and $\mu=4\left(m_{h}+p_{\perp}^{h h}\right)$ for the merged sample and $\mu=m_{h}$ and $\mu=4 m_{h}$ for the un-merged sample. The merging parameters were chosen to be $\bar{E}_{\text {Tclus }}=60 \mathrm{GeV}, \epsilon_{\text {clus }}=30 \mathrm{GeV}$. The ratio sub-plot is taken with respect to the un-merged sample with $\mu=2 m_{h}$.

the merged sample. More importantly, the scale variation in the observables $p_{\perp}^{h h}, p_{\perp}$ of the leading jet and $\Delta R(h, h)$, is substantially reduced with respect to the leading-order showered samples. This is particularly true in the regions where the parton shower is not expected to provide a good description of the additional radiation, namely in the $\Delta R(h, h) \lesssim \pi$ region and the high- $p_{\perp}$ regions. These improvements should not come as a surprise, as the considered observables are leading-order accurate for the merged sample in those regions, versus leading-logarithmic for the showered leading-order sample. The distribution of transverse momentum of a single Higgs boson, $p_{\perp}^{h}$, is not dominated by the extra radiation and thus the improvement is only marginal. 

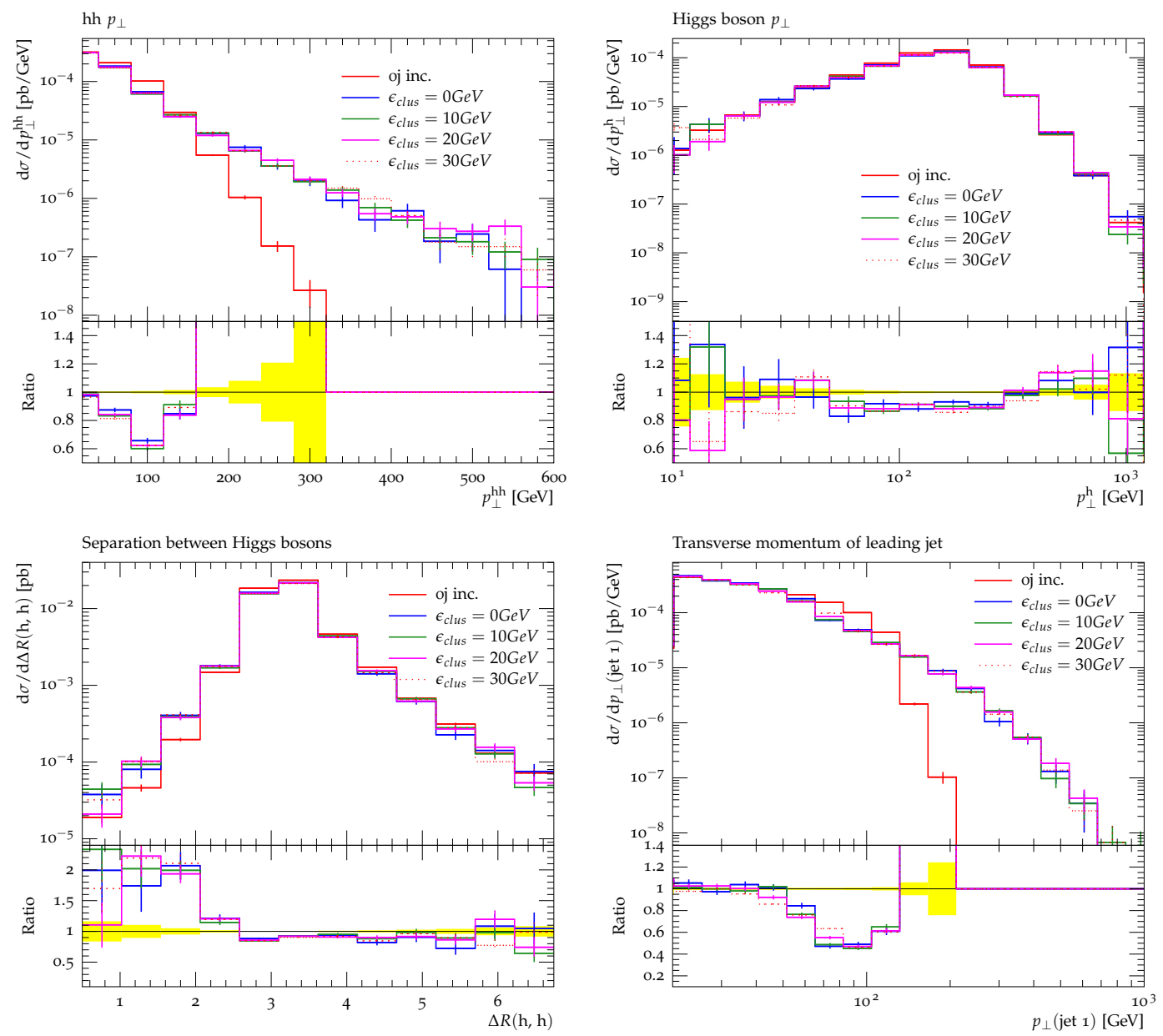

Figure 5. The transverse momentum of the di-Higgs system and the transverse momentum of a Higgs boson, $p_{\perp}^{h h}$ and $p_{\perp}^{h}$ respectively (top), the distance between the two Higgs bosons, $\Delta R(h, h)$, and the $p_{\perp}$ of the leading jet (bottom). Different $\epsilon_{\text {clus }}$ are chosen and the other parameters set to $\bar{E}_{\text {Tclus }}=60 \mathrm{GeV}, \mu=m_{h}+p_{\perp}^{h h}$. The ratio sub-plot is taken with respect to the un-merged sample with $\mu=m_{h}$ ('0j inc.') and the yellow bands in the ratio sub-plot represent the Monte Carlo statistical uncertainty in that sample.

In figure 5 we examine the effect of different choices of $\epsilon_{\text {clus }}$ in the range $[0,30] \mathrm{GeV}$. We again compare to the un-merged sample with $\mu=m_{h}$. The average merging scale was set to $\bar{E}_{\text {Tclus }}=60 \mathrm{GeV}$ and the scale $\mu$ was set to $\mu=m_{h}+p_{\perp}^{h h}$. Evidently, smoother merging of the samples can be achieved using higher values of $\epsilon_{\text {clus }}$.

In figure 6 we vary the average clustering scale, $\bar{E}_{\text {Tclus }}$, while keeping $\mu=m_{h}+p_{\perp}^{h h}$ and $\epsilon_{\text {clus }}=30 \mathrm{GeV}$. We again compare to the un-merged sample with $\mu=m_{h}$. Evidently, a relatively large systematic uncertainty comes from varying $\bar{E}_{\text {Tclus. }}$. This is due to the fact that changing $\bar{E}_{\text {Tclus }}$ alters the regions that the parton shower and matrix element calculation contribute in. For a lower $\bar{E}_{\text {Tclus }}$, the transition is smoother and we will use $\bar{E}_{\text {Tclus }}=50 \mathrm{GeV}$ for the phenomenological studies of the next section. We will contrast this to $\bar{E}_{\text {Tclus }}=70 \mathrm{GeV}$ where appropriate. 

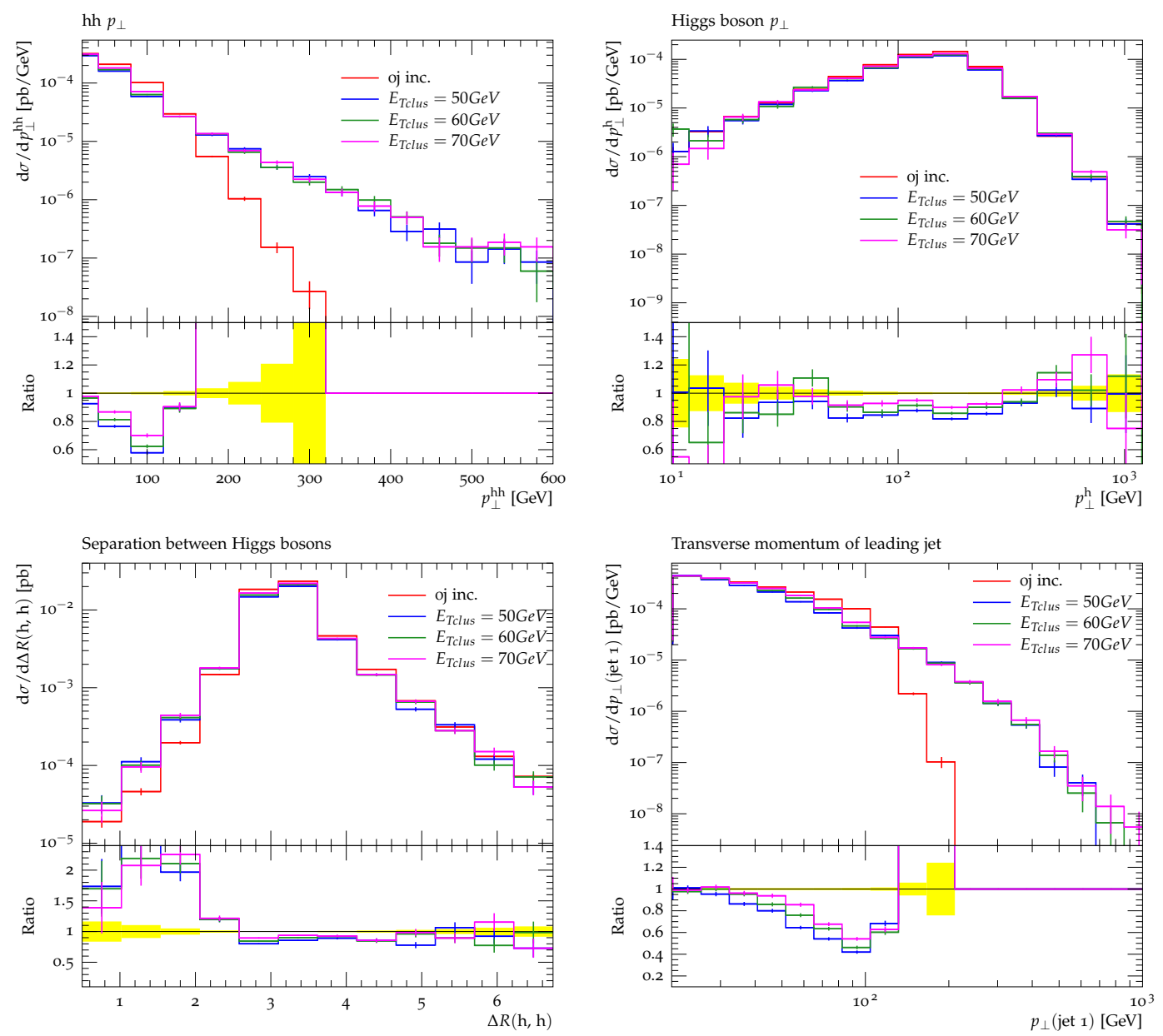

Figure 6. The transverse momentum of the di-Higgs system and the transverse momentum of a Higgs boson, $p_{\perp}^{h h}$ and $p_{\perp}^{h}$ respectively (top), the distance between the two Higgs bosons, $\Delta R(h, h)$, and the $p_{\perp}$ of the leading jet (bottom). Different $E_{\text {Tclus }}$ are chosen and the other parameters set to $\epsilon_{\text {clus }}=30 \mathrm{GeV}, \mu=m_{h}+p_{\perp}^{h h}$. The ratio sub-plot is taken with respect to the un-merged sample with $\mu=m_{h}$ ('0j inc.') and the yellow bands in the ratio sub-plot represent the Monte Carlo statistical uncertainty in that sample.

Finally, we show the envelope of the variation of both the merging scale $\bar{E}_{\text {Tclus }}$ (in $[50,70] \mathrm{GeV}$ ) and the variation scale $\epsilon_{\text {clus }}$ (in $[10,30] \mathrm{GeV}$ ) in figure 7 . In all of the samples, the scale was set to $\mu=m_{h}+p_{\perp}^{h h}$. The ratio sub-plot in the figure is taken with respect to the case where $E_{\text {Tclus }}=60 \mathrm{GeV}$ and $\epsilon_{\text {clus }}=20 \mathrm{GeV}$ and the error bars represent the Monte Carlo statistical uncertainty on that sample. For the $p_{\perp}^{h h}$ distribution, the uncertainty due to the variation of $E_{\text {Tclus }}$ and $\epsilon_{\text {clus }}$ is $\mathcal{O}(20 \%)$ up to $p_{\perp}^{h h} \sim 250 \mathrm{GeV}$ and grows to over $\sim 40 \%$ at higher values. The $p_{\perp}^{h}$ distribution exhibits variations of $\mathcal{O}(10 \%)$ or less up to $\sim 400 \mathrm{GeV}$ and down to $\sim 30 \mathrm{GeV}$. For the $\Delta R(h, h)$, the uncertainty is $\mathcal{O}(20 \%)$ in $\Delta R(h, h) \in[1.5,5]$ and close to $40 \%$ for $\Delta R(h, h) \sim 1$. Conclusions cannot be made for values outside this range since the samples are constrained by statistical fluctuations. The $p_{\perp}$ distribution of the hardest jet shows below $\mathcal{O}(10 \%)$ variations at low $p_{\perp}$, which grow to $\mathcal{O}(30 \%)$ around 

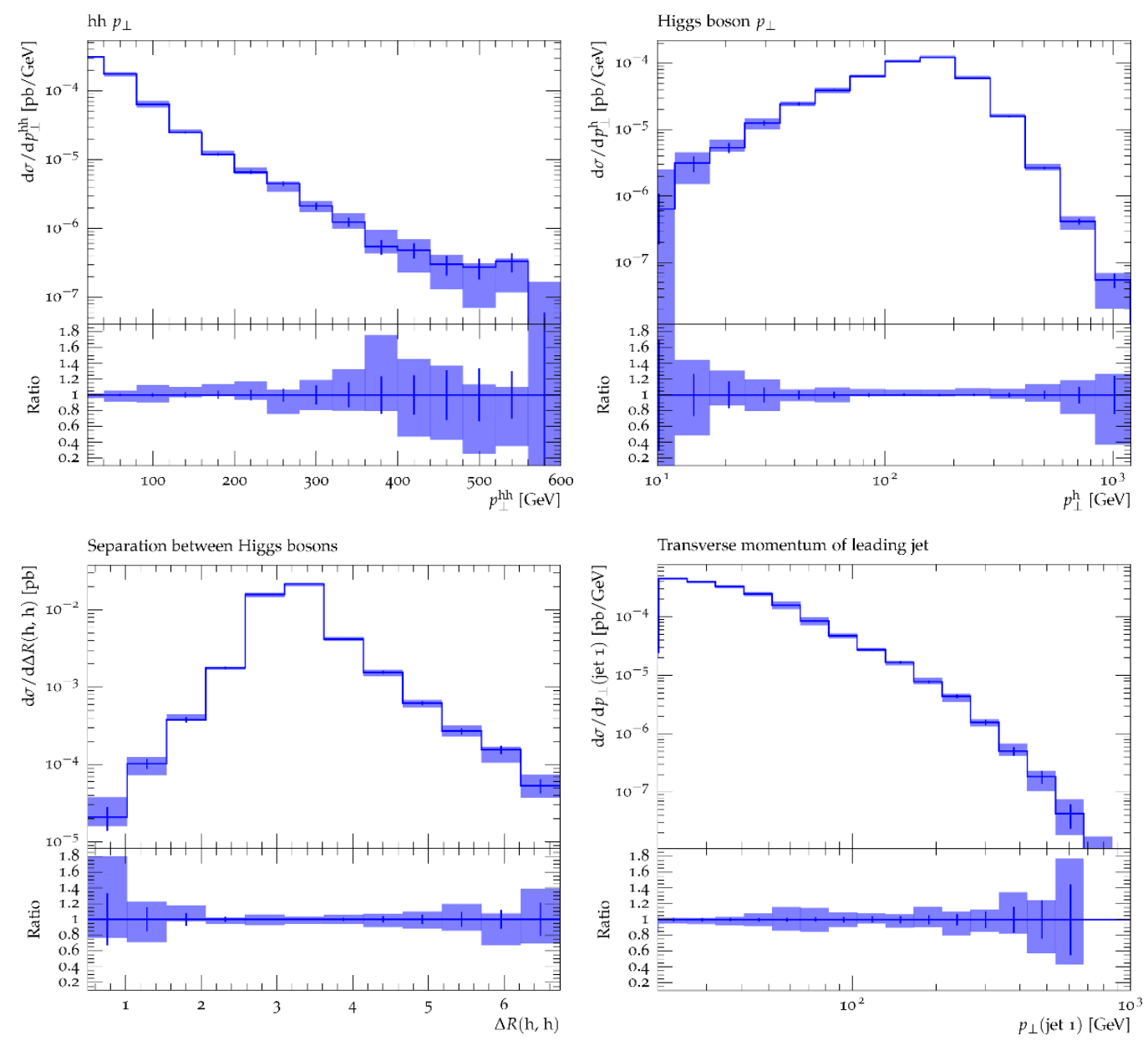

Figure 7. The transverse momentum of the di-Higgs system and the transverse momentum of a Higgs boson, $p_{\perp}^{h h}$ and $p_{\perp}^{h}$ respectively (top), the distance between the two Higgs bosons, $\Delta R(h, h)$ and the $p_{\perp}$ of the leading jet (bottom). The uncertainty envelope is constructed for $E_{\text {Tclus }} \in$ $[50,70] \mathrm{GeV}$ and $\epsilon_{\mathrm{clus}} \in[10,30] \mathrm{GeV}, \mu=m_{h}+p_{\perp}^{h h}$. The ratio sub-plot is taken with respect to the case where $E_{\text {Tclus }}=60 \mathrm{GeV}$ and $\epsilon_{\text {clus }}=20 \mathrm{GeV}$. The error bars represent the Monte Carlo statistical uncertainty for that set of parameters.

the region where the merging scale becomes significant, $[50,70] \mathrm{GeV}$, and are then reduced to $\mathcal{O}(10-20 \%)$ variations up to $p_{\perp} \sim 300 \mathrm{GeV}$.

\section{Phenomenological implications}

It is important to examine the implications of the merging on realistic phenomenological analyses of Higgs boson pair production at the LHC. We do this by focussing on an example of a decay channel with a relatively large branching ratio, $h h \rightarrow(b \bar{b})\left(\tau^{+} \tau^{-}\right)$. This has been examined in detail in $[14,15,30]$. We do not attempt here to perform a detailed signal versus background study; instead, we wish to show the magnitude of the effect of using the merged sample in a realistic analysis. We only focus on the top-anti-top background 
which will constitute the largest component of the irreducible background, via

$$
p p \rightarrow t \bar{t} \rightarrow\left(\tau^{-} \bar{\nu}_{\tau} b\right)\left(\tau^{+} \nu_{\tau} \bar{b}\right) .
$$

We consider the case of a $14 \mathrm{TeV}$ LHC, and normalise all $h h$ inclusive cross sections to the NNLO cross section obtained within the effective theory in [20], $\sigma_{h h}^{N N L O}=40.2 \mathrm{fb}$. We consider four different samples, un-merged with scales set to $\mu=m_{h}$ and $\mu=2 m_{h}$ and merged with scales $\mu=m_{h}+p_{\perp}^{h h}$ and $\mu=2\left(m_{h}+p_{\perp}^{h h}\right)$. The merging parameters were fixed to $E_{\text {Tclus }}=50 \mathrm{GeV}$ (or $70 \mathrm{GeV}$ ) and $\epsilon_{\text {clus }}=30 \mathrm{GeV}$. The $t \bar{t}$ background was generated via aMC@NLO $[63,64]$ along with the decays, and was assumed to have a total cross section of $\sigma_{t \bar{t}}=900 \mathrm{pb}[65,66]$. Showering and hadronization were performed using HERWIG++, and the simulation of the underlying event was included via multiple secondary parton interactions [67]. We follow the basic analysis steps as given in [30]: we assume $80 \% \tau$-reconstruction efficiency with negligible fake rate $^{6}$ and require two $\tau$-tagged jets with at least $p_{\perp}>20 \mathrm{GeV}$. We require that the taus, taken from the Monte Carlo truth, reproduce the Higgs mass within a $50 \mathrm{GeV}$ window, to account for the reconstruction smearing, as done in [30]. We use the Cambridge-Aachen jet algorithm available in the Fast Jet package $[68,69]$ with a radius parameter $R=1.4$ to search for so-called 'fat jets'. We require the existence of one fat jet in the event satisfying the mass-drop criteria as done in the $h V$ study in ref. [70]. We require the two hardest 'filtered' sub-jets to be b-tagged ${ }^{7}$ and to be central $(|\eta|<2.5)$ and the filtered fat jet to be in $\left(m_{h}-25 \mathrm{GeV}, m_{h}+25 \mathrm{GeV}\right)$. The b-tagging efficiency was taken to be $70 \%$, again with negligible fake rate for the sake of simplicity. We require a loose cut on the transverse momentum of the fat jet (after filtering) that satisfies the above criteria, $p_{\perp}^{\text {fat }}>100 \mathrm{GeV}$. This is done to maintain a sufficient number of events to examine the change of efficiencies with respect to other cuts. We also apply a transverse momentum cut on the $\tau^{+} \tau^{-}$system of equal magnitude, $p_{\perp}^{\tau \tau}>100 \mathrm{GeV}$.

We wish to examine the stability of the merged samples against that of the un-merged samples with respect to scale variations. It is obvious that sufficiently inclusive quantities should not differ in a way that will impact the analyses. However, there are quantities for which the merged sample and the un-merged sample differ substantially. As an exercise, we examine two such observables here: the distance between the $\left(\tau^{+} \tau^{-}\right)$system and the $(b \bar{b})$ system (equivalent to the distance between the Higgs bosons), and the transverse momentum of the $\tau^{+} \tau^{-} b \bar{b}$ system (equivalent to the transverse momentum of the the $h h$ system). Figure 8 demonstrates that it is conceivable that both of these observables may be used for background rejections. Moreover, it is evident that the largest uncertainties in the $h h$ signal predictions are present in the exact same region that one would wish to place the cuts in: $p_{T}(h h) \sim 100 \mathrm{GeV}$ and $\Delta R(h, h) \sim 3$. What is also important is the fact that both the 0 -jet exclusive and the 1-jet inclusive signal samples contribute in the region of interest, as demonstrated in figure 9 for the case where $\mu=m_{h}+p_{\perp}^{h h}$.

We can further quantify the effect observed in figure 8 . The stability of the samples can be assessed by taking ratios of the efficiencies for different cut values. If the efficiency does

\footnotetext{
${ }^{6}$ Thus, we do not consider any mistagging backgrounds, which could be potentially important.

${ }^{7}$ Bottom-jet tagging was performed by setting the bottom mesons to stable in the HERWIG++ event generator.
} 

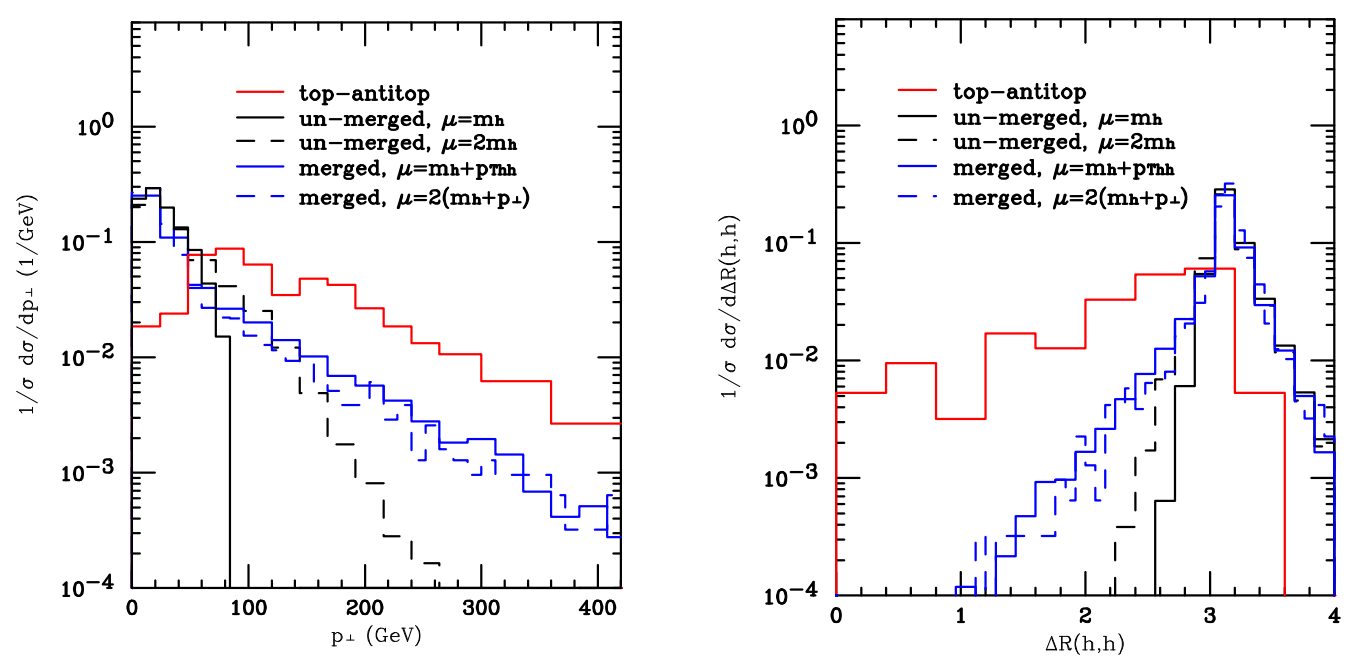

Figure 8. The reconstructed transverse momentum of the Higgs boson pair (left) and the distance between the reconstructed Higgs bosons (right) resulting from the analysis outlined in the main text for the different signal samples (merged or un-merged) and the top-anti-top background.
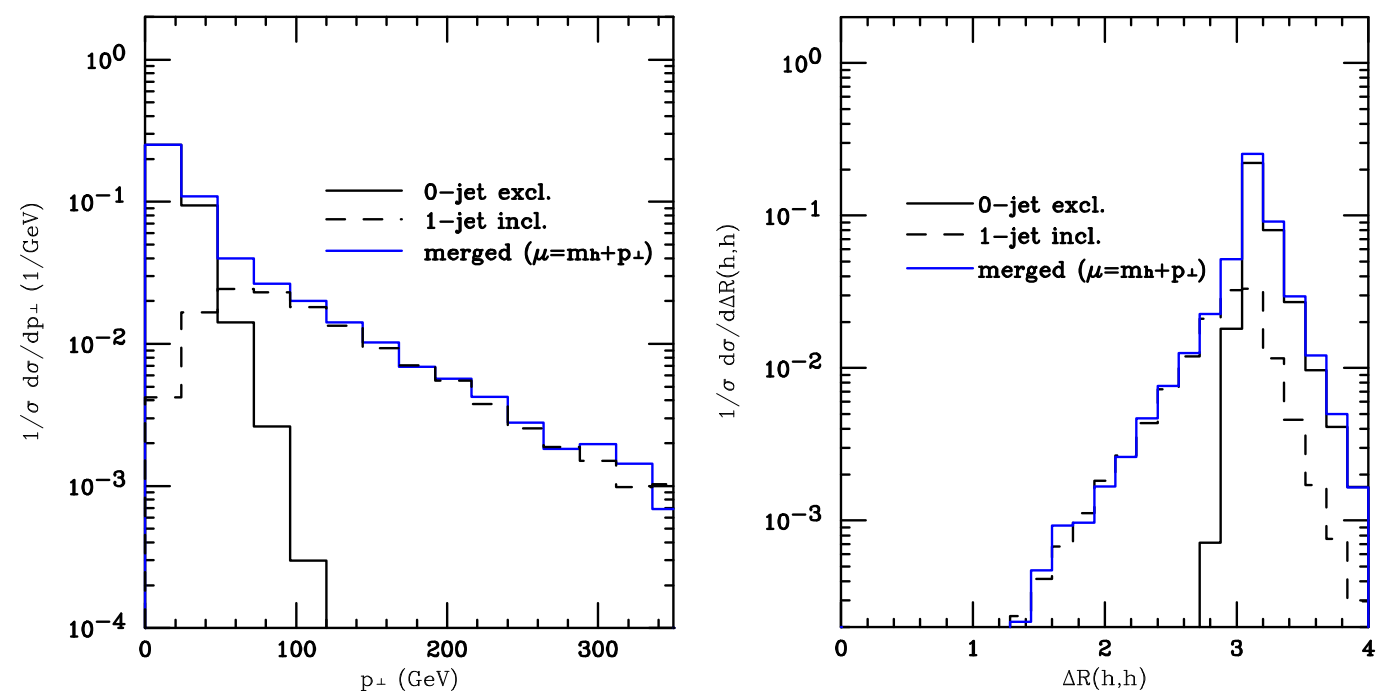

Figure 9. The reconstructed transverse momentum of the Higgs boson pair (left) and the distance between the reconstructed Higgs bosons (right) resulting from the analysis outlined in the main text broken into their individual 0 -jet exclusive and 1-jet inclusive contributions. The scale was chosen to be $\mu=m_{h}+p_{\perp}^{h h}$.

not vary substantially with the cuts, then the sample (merged or un-merged) is deemed to be stable, and the theoretical systematic uncertainty on the efficiency of the cut can be considered to be low. Figure 10 shows the variation of the ratio of efficiencies for different parameters for the merged and un-merged samples:

$$
R=\text { (cut efficiency, sample } \mathrm{i}) /(\text { cut efficiency, sample } \mathrm{j} \text { ) }
$$

for cuts on the aforementioned observables which we abbreviate as $p_{\perp, \max }$ and $\Delta R_{\min }(h, h)$. 

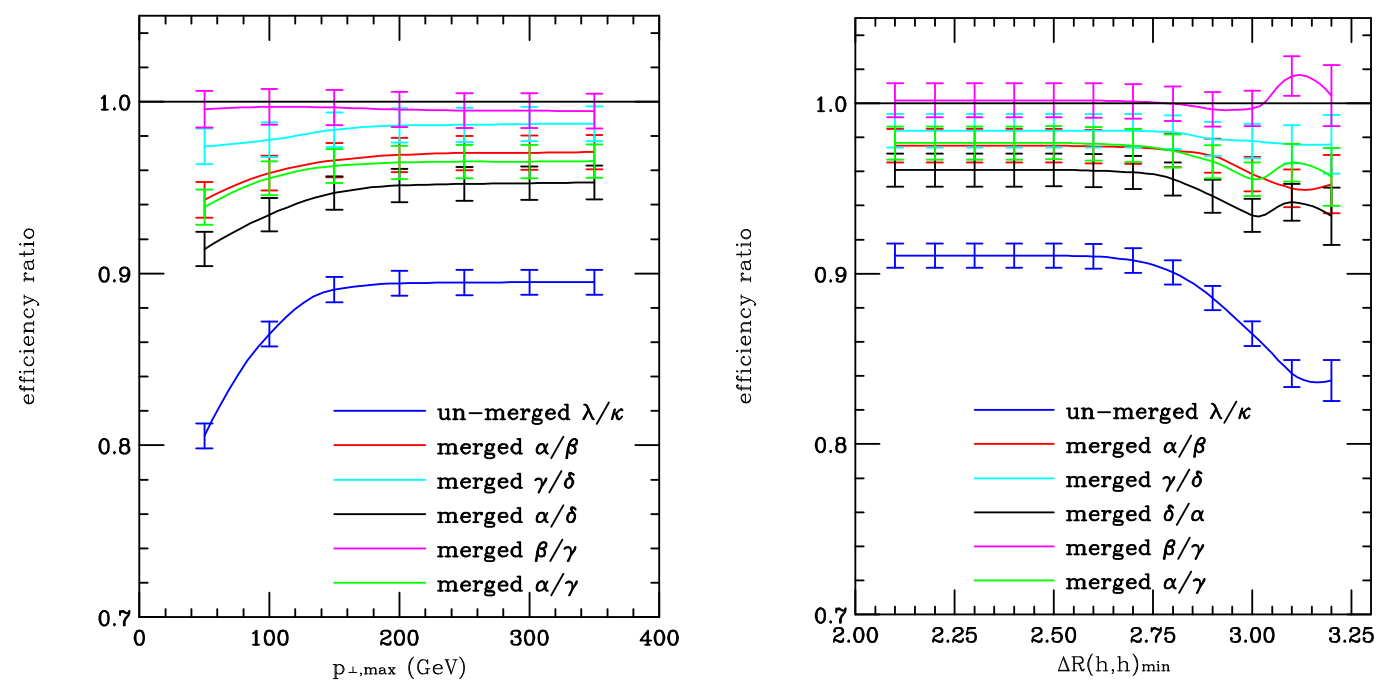

Figure 10. The variation of the ratio of efficiencies with different values of the cuts $p_{\perp, \max }$ (left) and $\Delta R_{\min }(h, h)$ (right) between two different samples for merged and un-merged samples. The sample parameters are: un-merged: $\kappa: \mu=m_{h}, \lambda: \mu=2 m_{h}$. merged with $\epsilon_{\text {clus }}=30 \mathrm{GeV}: \alpha$ : $\left(\mu=m_{h}+p_{\perp}^{h h}, E_{\text {Tclus }}=50 \mathrm{GeV}\right), \beta:\left(\mu=2\left(m_{h}+p_{\perp}^{h h}\right), E_{\text {Tclus }}=50 \mathrm{GeV}\right), \gamma:\left(\mu=m_{h}+p_{\perp}^{h h}\right.$, $\left.E_{\text {Tclus }}=70 \mathrm{GeV}\right), \delta:\left(\mu=2\left(m_{h}+p_{\perp}^{h h}\right), E_{\text {Tclus }}=70 \mathrm{GeV}\right)$, all with $\epsilon_{\text {clus }}=30 \mathrm{GeV}$.

For details of the parameters used for each of the samples $\{\alpha, \beta, \gamma, \delta, \iota, \kappa\}$, see the caption of figure 10. The un-merged samples $\{\kappa, \lambda\}$ exhibit a fairly substantial change in the ratio of efficiencies for the two chosen scales, starting from $10 \%$ and going up to $\sim 20 \%$ for some values of the cuts. This change can be interpreted as a theoretical systematic uncertainty on the efficiency itself. The merged samples $\{\alpha, \beta, \gamma, \delta\}$ perform better, with lower overall variation of the efficiency ratio, with the deviations always $<10 \%$ as demonstrated in the figure 10, while, more importantly, on average possessing an efficiency variation of $\sim 5 \%$. The differences are due to the fact that the chosen observables are sensitive to the behaviour of the extra radiation, which, at high transverse momentum or large separations between the Higgs bosons, is not predicted reliably by the parton shower. ${ }^{8}$

For completeness, in table 2 we show a set of cuts and resulting cross sections resulting from the analysis. We provide also explicit cuts on the variables we have examined: $\Delta R(h, h)>2.8$ and $p_{\perp}^{h h}<80 \mathrm{GeV}$. The final result for this basic analysis is optimistic, with $S / B \sim 0.4-0.5$ for all samples, leading to a reasonable significance at $600 \mathrm{fb}^{-1}$ of integrated luminosity, expected to be collected at the LHC in the full phase of Run II. It is worthwhile to note that the final cross section prediction for the merged samples $(\alpha$ and $\beta$ ) after all cuts only exhibits a $\sim 2 \%$ variation compared to the un-merged samples $(\kappa$ and $\lambda$ ) which exhibit a $\sim 13 \%$ variation.

\footnotetext{
${ }^{8}$ The ratio differs from unity since we expect differences in response to cuts in other observables between the two samples. This can also be seen in the cut flows of table 2. Nevertheless, the merged sample ratios are still closer to unity overall than those of the un-merged samples, demonstrating further the increased accuracy of the calculation.
} 


\begin{tabular}{|c|c|c|c|c|c|c|c|c|c|}
\hline Process & $\kappa$ & $\lambda$ & $\alpha$ & $\beta$ & $t \bar{t}$ & $S / B(\kappa)$ & $S / B(\lambda)$ & $S / B(\alpha)$ & $S / B(\beta)$ \\
\hline$\sigma[\mathrm{fb}]$ & 40.20 & 40.20 & 40.20 & 40.20 & $9 \times 10^{5}$ & .00004 & .00004 & .00004 & .00004 \\
\hline BRs & 2.97 & 2.97 & 2.97 & 2.97 & 11000 & .00027 & .00027 & .00027 & .00027 \\
\hline$\tau$ cuts & 0.78 & 0.82 & 0.79 & 0.80 & 296.4 & .00263 & .00277 & .00266 & .00270 \\
\hline fat jet cuts & 0.106 & 0.104 & 0.11 & 0.11 & 0.93 & 0.11 & 0.11 & 0.12 & 0.12 \\
\hline$\Delta R(h, h)$ & 0.106 & 0.100 & 0.099 & 0.101 & 0.310 & 0.34 & 0.32 & 0.32 & 0.33 \\
\hline$p_{\perp}^{h h}$ & 0.103 & 0.089 & 0.095 & 0.093 & 0.207 & 0.50 & 0.43 & 0.46 & 0.45 \\
\hline
\end{tabular}

Table 2. Cross sections for the $h h$ signal and $t \bar{t}$ aMC@NLO background after series of cuts. The un-merged samples $\kappa$ and $\lambda$ have $\mu=m_{h}$ and $\mu=2 m_{h}$ respectively and the merged signal samples ' $\alpha$ ' and ' $\beta$ ' have $\mu=m_{h}+p_{\perp}^{h h}$ and $\mu=2\left(m_{h}+p_{\perp}^{h h}\right)$ respectively, as well as $E_{\text {Tclus }}=50 \mathrm{GeV}$ and $\epsilon_{\text {clus }}=30 \mathrm{GeV}$. The final two cuts were chosen to be $\Delta R(h, h)>2.8$ and $p_{\perp}^{h h}<80 \mathrm{GeV}$.

\section{Conclusions}

We have described the implementation of Higgs boson pair production merged to the onejet matrix elements, generated using OpenLoops, in the HERWIG++ event generator. We have examined the systematic uncertainties associated with the merging. Moreover, we have provided examples of the magnitude of the effects of using the merged samples in a realistic analysis. As was demonstrated in this analysis, using the leading order matrix elements in conjunction with the parton shower can potentially introduce $\mathcal{O}(20 \%)$ systematic uncertainties in the predictions of the efficiencies of experimental cuts. The uncertainty will inexorably propagate to measurements of the Higgs boson self-coupling. The merged samples demonstrate theoretical uncertainties on the efficiencies that are $10 \%$ or better for the examined observables. We expect such conclusions to remain valid for a future NLO simulation matched to the parton shower. We thus recommend the use of samples that include the merged exact one-jet matrix elements in all future phenomenological or experimental analyses of the process. The Monte Carlo event generator developed for this project is available as an add-on to the HERWIG++ event generator at http://www.physik.uzh.ch/data/openloops/download/projects/hhmerge/.

\section{Acknowledgments}

We would like to thank Thomas Gehrmann, Massimiliano Grazzini, Stefano Pozzorini, José Zurita and Paolo Torrielli for constructive discussions during the project. This research is supported in part by the Swiss National Science Foundation (SNF) under contract 200020149517 and by the European Commission through the "LHCPhenoNet" Initial Training Network PITN-GA-2010-264564.

Open Access. This article is distributed under the terms of the Creative Commons Attribution License (CC-BY 4.0), which permits any use, distribution and reproduction in any medium, provided the original author(s) and source are credited. 


\section{References}

[1] ATLAS collaboration, Observation of a new particle in the search for the standard model Higgs boson with the ATLAS detector at the LHC, Phys. Lett. B 716 (2012) 1 [arXiv:1207.7214] [INSPIRE].

[2] CMS collaboration, Observation of a new boson at a mass of $125 \mathrm{GeV}$ with the CMS experiment at the LHC, CMS-HIG-12-028 (2012).

[3] CMS collaboration, Combination of standard model Higgs boson searches and measurements of the properties of the new boson with a mass near $125 \mathrm{GeV}$, CMS-PAS-HIG-12-045 (1494149).

[4] ATLAS collaboration, Coupling properties of the new Higgs-like boson observed with the ATLAS detector at the LHC, ATLAS-CONF-2012-127 (2012).

[5] ATLAS collaboration, An update of combined measurements of the new Higgs-like boson with high mass resolution channels, ATLAS-CONF-2012-170 (2012).

[6] T. Plehn and M. Rauch, The quartic Higgs coupling at hadron colliders, Phys. Rev. D 72 (2005) 053008 [hep-ph/0507321] [INSPIRE].

[7] T. Binoth, S. Karg, N. Kauer and R. Ruckl, Multi-Higgs boson production in the Standard Model and beyond, Phys. Rev. D 74 (2006) 113008 [hep-ph/0608057] [InSPIRE].

[8] E.N. Glover and J. van der Bij, Higgs boson pair production via gluon fusion, Nucl. Phys. B 309 (1988) 282 [INSPIRE].

[9] S. Dawson, S. Dittmaier and M. Spira, Neutral Higgs boson pair production at hadron colliders: QCD corrections, Phys. Rev. D 58 (1998) 115012 [hep-ph/9805244] [INSPIRE].

[10] A. Djouadi, W. Kilian, M. Muhlleitner and P. Zerwas, Production of neutral Higgs boson pairs at LHC, Eur. Phys. J. C 10 (1999) 45 [hep-ph/9904287] [INSPIRE].

[11] T. Plehn, M. Spira and P. Zerwas, Pair production of neutral Higgs particles in gluon-gluon collisions, Nucl. Phys. B 479 (1996) 46 [Erratum ibid. B 531 (1998) 655] [hep-ph/9603205] [INSPIRE].

[12] U. Baur, T. Plehn and D.L. Rainwater, Determining the Higgs boson selfcoupling at hadron colliders, Phys. Rev. D 67 (2003) 033003 [hep-ph/0211224] [INSPIRE].

[13] U. Baur, T. Plehn and D.L. Rainwater, Probing the Higgs selfcoupling at hadron colliders using rare decays, Phys. Rev. D 69 (2004) 053004 [hep-ph/0310056] [INSPIRE].

[14] J. Baglio et al., The measurement of the Higgs self-coupling at the LHC: theoretical status, JHEP 04 (2013) 151 [arXiv: 1212.5581] [INSPIRE].

[15] A.J. Barr, M.J. Dolan, C. Englert and M. Spannowsky, Di-Higgs final states augMT2ed Selecting hh events at the high luminosity LHC, Phys. Lett. B 728 (2014) 308 [arXiv: 1309.6318] [INSPIRE].

[16] M.J. Dolan, C. Englert, N. Greiner and M. Spannowsky, Further on up the road: hhjj production at the LHC, arXiv:1310.1084 [INSPIRE].

[17] A. Papaefstathiou, L.L. Yang and J. Zurita, Higgs boson pair production at the LHC in the $b \bar{b} W^{+} W^{-}$channel, Phys. Rev. D 87 (2013) 011301 [arXiv:1209.1489] [InSPIRE].

[18] F. Goertz, A. Papaefstathiou, L.L. Yang and J. Zurita, Higgs boson self-coupling measurements using ratios of cross sections, JHEP 06 (2013) 016 [arXiv:1301.3492] [INSPIRE]. 
[19] F. Goertz, A. Papaefstathiou, L.L. Yang and J. Zurita, Measuring the Higgs boson self-coupling at the LHC using ratios of cross sections, arXiv:1309.3805 [INSPIRE].

[20] D. de Florian and J. Mazzitelli, Higgs boson pair production at next-to-next-to-leading order in QCD, Phys. Rev. Lett. 111, 201801 (2013) 201801 [arXiv: 1309.6594] [INSPIRE].

[21] D. de Florian and J. Mazzitelli, Two-loop virtual corrections to Higgs pair production, Phys. Lett. B 724 (2013) 306 [arXiv:1305.5206] [INSPIRE].

[22] J. Grigo, J. Hoff, K. Melnikov and M. Steinhauser, On the Higgs boson pair production at the LHC, Nucl. Phys. B 875 (2013) 1 [arXiv:1305.7340] [INSPIRE].

[23] J. Cao, Z. Heng, L. Shang, P. Wan and J.M. Yang, Pair production of a $125 \mathrm{GeV}$ Higgs boson in MSSM and NMSSM at the LHC, JHEP 04 (2013) 134 [arXiv: 1301.6437] [INSPIRE].

[24] R.S. Gupta, H. Rzehak and J.D. Wells, How well do we need to measure the Higgs boson mass and self-coupling?, Phys. Rev. D 88 (2013) 055024 [arXiv:1305.6397] [InSPIRE].

[25] D.T. Nhung, M. Muhlleitner, J. Streicher and K. Walz, Higher order corrections to the trilinear Higgs self-couplings in the real NMSSM, JHEP 11 (2013) 181 [arXiv:1306.3926] [INSPIRE].

[26] U. Ellwanger, Higgs pair production in the NMSSM at the LHC, JHEP 08 (2013) 077 [arXiv: 1306.5541] [INSPIRE].

[27] J.M. No and M. Ramsey-Musolf, Probing the Higgs portal at the LHC through resonant di-Higgs production, arXiv:1310.6035 [INSPIRE].

[28] M. McCullough, A new indirect probe of the Higgs self-coupling, arXiv:1312.3322 [INSPIRE].

[29] Q. Li, Q.-S. Yan and X. Zhao, Higgs pair production: improved description by matrix element matching, Phys. Rev. D 89 (2014) 033015 [arXiv:1312.3830] [INSPIRE].

[30] M.J. Dolan, C. Englert and M. Spannowsky, Higgs self-coupling measurements at the LHC, JHEP 10 (2012) 112 [arXiv:1206.5001] [INSPIRE].

[31] S. Dawson, E. Furlan and I. Lewis, Unravelling an extended quark sector through multiple Higgs production?, Phys. Rev. D 87 (2013) 014007 [arXiv:1210.6663] [INSPIRE].

[32] S. Dawson, S. Dittmaier and M. Spira, Two loop QCD corrections to Higgs pair production at the LHC, Acta Phys. Polon. B 29 (1998) 2875 [hep-ph/9806304] [INSPIRE].

[33] CMS collaboration, Jet production rates in association with $W$ and $Z$ bosons in pp collisions at $\sqrt{s}=7$ TeV, JHEP 01 (2012) 010 [arXiv:1110.3226] [INSPIRE].

[34] ATLAS collaboration, Study of jets produced in association with a $W$ boson in pp collisions at $\sqrt{s}=7$ TeV with the ATLAS detector, Phys. Rev. D 85 (2012) 092002 [arXiv:1201.1276] [INSPIRE].

[35] F. Cascioli, P. Maierhofer and S. Pozzorini, Scattering amplitudes with open loops, Phys. Rev. Lett. 108 (2012) 111601 [arXiv:1111.5206] [INSPIRE].

[36] A. Denner and S. Dittmaier, Reduction of one loop tensor five point integrals, Nucl. Phys. B 658 (2003) 175 [hep-ph/0212259] [INSPIRE].

[37] A. Denner and S. Dittmaier, Reduction schemes for one-loop tensor integrals, Nucl. Phys. B 734 (2006) 62 [hep-ph/0509141] [INSPIRE].

[38] A. Denner and S. Dittmaier, Scalar one-loop 4-point integrals, Nucl. Phys. B 844 (2011) 199 [arXiv: 1005.2076] [INSPIRE].

[39] F. Cascioli et al., Precise Higgs-background predictions: merging NLO QCD and squared quark-loop corrections to four-lepton + 0,1 jet production, JHEP 01 (2014) 046 [arXiv: 1309.0500] [INSPIRE]. 
[40] T. Gleisberg et al., Event generation with SHERPA 1.1, JHEP 02 (2009) 007 [arXiv:0811.4622] [INSPIRE].

[41] A. Martin, W. Stirling, R. Thorne and G. Watt, Parton distributions for the LHC, Eur. Phys. J. C 63 (2009) 189 [arXiv:0901.0002] [InSPIRE].

[42] A. Martin, W. Stirling, R. Thorne and G. Watt, Uncertainties on $\alpha_{s}$ in global PDF analyses and implications for predicted hadronic cross sections, Eur. Phys. J. C 64 (2009) 653 [arXiv: 0905.3531] [INSPIRE].

[43] A. Martin, W. Stirling, R. Thorne and G. Watt, Heavy-quark mass dependence in global PDF analyses and 3- and 4-flavour parton distributions, Eur. Phys. J. C 70 (2010) 51 [arXiv: 1007.2624] [INSPIRE].

[44] M.L. Mangano, M. Moretti and R. Pittau, Multijet matrix elements and shower evolution in hadronic collisions: $W b \bar{b}+n$ jets as a case study, Nucl. Phys. B 632 (2002) 343 [hep-ph/0108069] [INSPIRE].

[45] M. Mangano, Merging multijet matrix elements and shower evolution in hadronic collisions, http://mlm.web.cern.ch/mlm/talks/lund-alpgen.pdf (2004).

[46] J. Alwall et al., Comparative study of various algorithms for the merging of parton showers and matrix elements in hadronic collisions, Eur. Phys. J. C 53 (2008) 473 [arXiv:0706.2569] [INSPIRE].

[47] S. Catani, F. Krauss, R. Kuhn and B. Webber, QCD matrix elements + parton showers, JHEP 11 (2001) 063 [hep-ph/0109231] [INSPIRE].

[48] L. Lönnblad, Correcting the color dipole cascade model with fixed order matrix elements, JHEP 05 (2002) 046 [hep-ph/0112284] [INSPIRE].

[49] F. Krauss, Matrix elements and parton showers in hadronic interactions, JHEP 08 (2002) 015 [hep-ph/0205283] [INSPIRE].

[50] S. Mrenna and P. Richardson, Matching matrix elements and parton showers with HERWIG and PYTHIA, JHEP 05 (2004) 040 [hep-ph/0312274] [INSPIRE].

[51] P. Nason, A New method for combining NLO QCD with shower Monte Carlo algorithms, JHEP 11 (2004) 040 [hep-ph/0409146] [INSPIRE].

[52] S. Frixione, P. Nason and C. Oleari, Matching NLO QCD computations with Parton Shower simulations: the POWHEG method, JHEP 11 (2007) 070 [arXiv:0709.2092] [INSPIRE].

[53] S. Frixione, F. Stoeckli, P. Torrielli, B.R. Webber and C.D. White, The MCaNLO 4.0 event generator, arXiv: 1010.0819 [INSPIRE].

[54] S. Frixione and B.R. Webber, Matching NLO QCD computations and parton shower simulations, JHEP 06 (2002) 029 [hep-ph/0204244] [INSPIRE].

[55] S. Gieseke et al., HERWIG++ 2.5 release note, arXiv:1102.1672 [INSPIRE].

[56] K. Arnold et al., HERWIG++ 2.6 release note, arXiv:1205.4902 [INSPIRE].

[57] M. Bahr et al., HERWIG++ physics and manual, Eur. Phys. J. C 58 (2008) 639 [arXiv:0803.0883] [INSPIRE].

[58] J. Bellm et al., HERWIG++ 2.7 release note, arXiv:1310.6877 [INSPIRE].

[59] G. Corcella et al., HERWIG 6: an event generator for hadron emission reactions with interfering gluons (including supersymmetric processes), JHEP 01 (2001) 010 [hep-ph/0011363] [INSPIRE]. 
[60] R. Frederix and S. Frixione, Merging meets matching in MC@NLO, JHEP 12 (2012) 061 [arXiv: 1209.6215] [INSPIRE].

[61] A. Buckley et al., Rivet user manual, Comput. Phys. Commun. 184 (2013) 2803 [arXiv: 1003.0694] [INSPIRE].

[62] M. Cacciari, G.P. Salam and G. Soyez, The anti- $k_{t}$ jet clustering algorithm, JHEP 04 (2008) 063 [arXiv: 0802.1189] [INSPIRE].

[63] S. Frixione, F. Stoeckli, P. Torrielli and B.R. Webber, NLO QCD corrections in HERWIG++ with MC@NLO, JHEP 01 (2011) 053 [arXiv: 1010.0568] [INSPIRE].

[64] R. Frederix et al., Scalar and pseudoscalar Higgs production in association with a top-antitop pair, Phys. Lett. B 701 (2011) 427 [arXiv:1104.5613] [INSPIRE].

[65] V. Ahrens, A. Ferroglia, M. Neubert, B.D. Pecjak and L.L. Yang, Precision predictions for the $t+\bar{t}$ production cross section at hadron colliders, Phys. Lett. B 703 (2011) 135 [arXiv: 1105.5824] [INSPIRE].

[66] M. Czakon, P. Fiedler and A. Mitov, The total top quark pair production cross-section at hadron colliders through $O\left(\alpha_{S}^{4}\right)$, Phys. Rev. Lett. 110 (2013) 252004 [arXiv:1303.6254] [INSPIRE].

[67] M. Bahr, S. Gieseke and M.H. Seymour, Simulation of multiple partonic interactions in HERWIG++, JHEP 07 (2008) 076 [arXiv:0803.3633] [INSPIRE].

[68] M. Cacciari, G.P. Salam and G. Soyez, FastJet user manual, Eur. Phys. J. C 72 (2012) 1896 [arXiv: 1111.6097] [INSPIRE].

[69] M. Cacciari and G.P. Salam, Dispelling the $N^{3}$ myth for the $k_{t}$ jet-finder, Phys. Lett. B 641 (2006) 57 [hep-ph/0512210] [INSPIRE].

[70] J.M. Butterworth, A.R. Davison, M. Rubin and G.P. Salam, Jet substructure as a new Higgs search channel at the LHC, Phys. Rev. Lett. 100 (2008) 242001 [arXiv:0802.2470] [INSPIRE]. 